\title{
Automatic Gauge Control in Rolling Process Based on Multiple Smith Predictor Models
}

\author{
Jiangyun $\mathrm{Li},{ }^{1}$ Kang Wang, ${ }^{1}$ and Yang $\mathrm{Li}^{2}$ \\ ${ }^{1}$ School of Automation and Electrical Engineering, University of Science and Technology Beijing, Beijing 100083, China \\ ${ }^{2}$ School of International Studies, Communication University of China (CUC), Beijing 100024, China \\ Correspondence should be addressed to Kang Wang; vangkang@163.com
}

Received 13 August 2014; Accepted 12 September 2014; Published 24 November 2014

Academic Editor: Shen Yin

Copyright (c) 2014 Jiangyun Li et al. This is an open access article distributed under the Creative Commons Attribution License, which permits unrestricted use, distribution, and reproduction in any medium, provided the original work is properly cited.

\begin{abstract}
Automatic rolling process is a high-speed system which always requires high-speed control and communication capabilities. Meanwhile, it is also a typical complex electromechanical system; distributed control has become the mainstream of computer control system for rolling mill. Generally, the control system adopts the 2-level control structure-basic automation (Level 1) and process control (Level 2) - to achieve the automatic gauge control. In Level 1, there is always a certain distance between the roll gap of each stand and the thickness testing point, leading to the time delay of gauge control. Smith predictor is a method to cope with time-delay system, but the practical feedback control based on traditional Smith predictor cannot get the ideal control result, because the time delay is hard to be measured precisely and in some situations it may vary in a certain range. In this paper, based on adaptive Smith predictor, we employ multiple models to cover the uncertainties of time delay. The optimal model will be selected by the proposed switch mechanism. Simulations show that the proposed multiple Smith model method exhibits excellent performance in improving the control result even for system with jumping time delay.
\end{abstract}

\section{Introduction}

Because the control objects are electromechanical and hydraulic systems with small inertia and fast response, automatic rolling is a fast process which requires high-speed control and communication capabilities. With the technological and industrial development, requirements for high-quality strips and high-level automation are becoming critical. Distributed control has become the mainstream of computer control system for rolling mill which is a typical complex electromechanical system $[1,2]$. Generally, the control system adopts the 2-level control structure-basic automation (Level 1) and process control (Level 2)-to achieve automatic gauge control $[3,4]$. Composed of process server and high-speed communication network, the process control (Level 2) can accomplish mathematical model calculating, initial values setting, and material tracking. This level always has a high demand on network bandwidth with the ability to transfer, store, and share massive process information; a typical network adopted is the industrial Ethernet. Material tracking and initial value settings of roll gap and roll force in finish rolling AGC (automatic gauge control) process are calculated through the mathematical model by the servers in Level 2; corresponding information is transferred to the basic automation controllers through Ethernet, shown as in Figure 1. The basic automation Level 1 accomplishes the fast collection of process information and the output of control results; controllers in this level are high-speed embedded controllers or high-performance PLC. Because of the time requirement of fast loop control, network communication rates among controllers are also highly demanded. Typical networks adopted in this level are the GE's RFnet (reflective memory network) and Siemens' GDM (global data memory) network. Figure 2 shows the basic automation network deployment of a tandem rolling line, in which controllers in the rough rolling section, finish rolling section, and basic automation are connected through RFnet. In conclusion, network delay and packet loss in AGC will both influence the manufacturing process and even lead to the product disqualified in severe cases. 


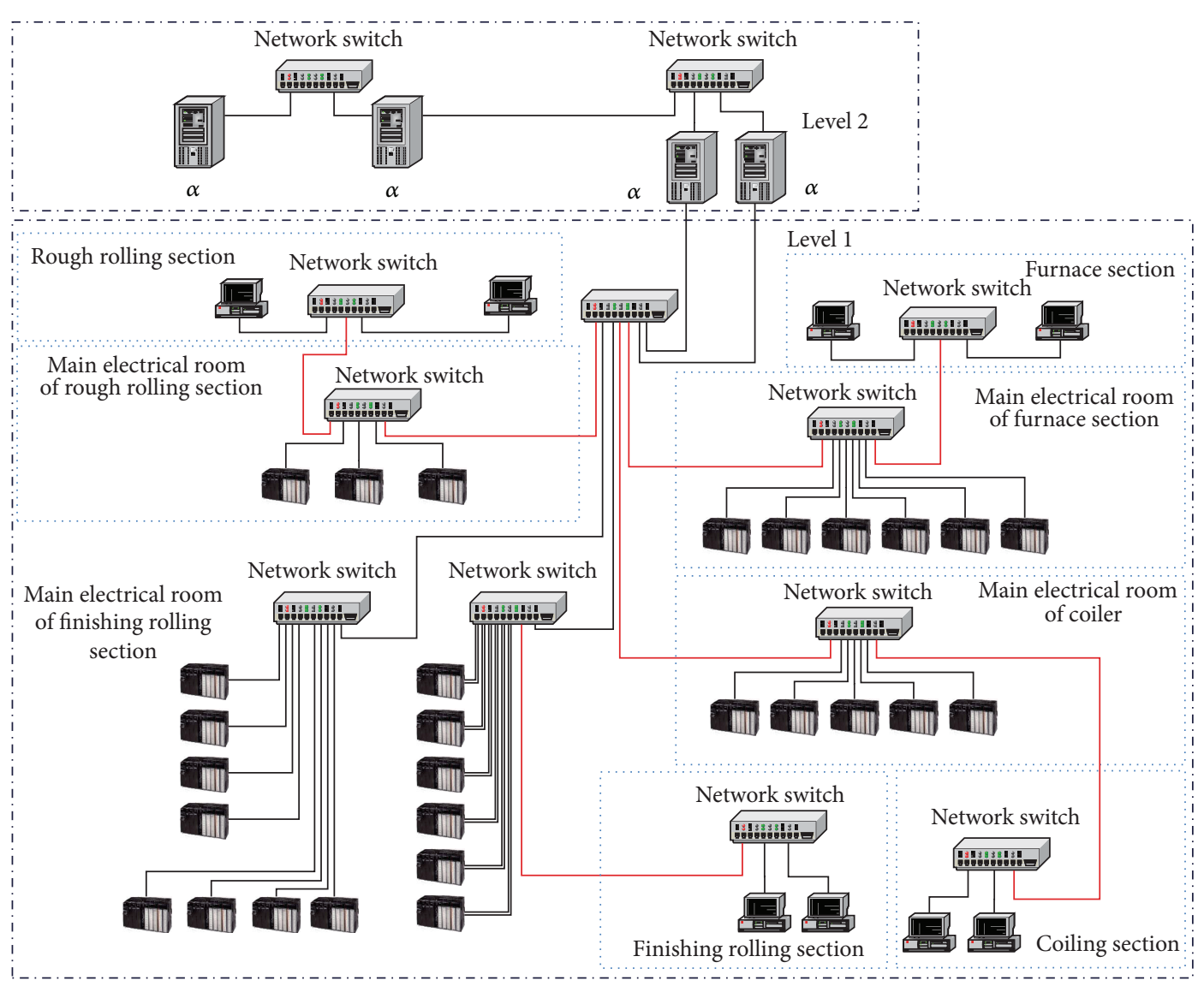

Hot rolling Ethernet

— Armored fiber

— Twisted pair

- Twisted pair (2)

FIGURE 1: Ethernet network structure in a tandem rolling line.

In practical AGC thickness control process, two main factors will lead to the uncertain time delay for controlled system. One is the certain distance between the thickness gauge and rolling gap of each stand, and the other is the essentially existing time delay in network transmission. Smith predictor is a method to deal with above problem, but as the value of system time delay is hard to be measured precisely, the Smith predictor method always cannot be carried out effectively in practice. Adaptive Smith predictor model can cope with partial model mismatch $[5,6]$. However, improper selection of adaptive initial value may also cause the system response with bad transient process; this situation may get worse for system with variant time delay because the single predictor cannot match all conditions with different time delay well. Multiple model adaptive control (MMAC) is a kind of tool for adaptive control where multiple element models will be set up to cover the uncertainty of the parameter or structure of the system, and a switching mechanism will be used to form the final multiple model controller. This kind of controller can be used to improve the transient response [7]. From the 1990s, a lot of MMAC based on continuous-time $[8,9]$ and discretetime [10] system have been given, and a lot of application of MMAC also can be seen [11, 12].

In this paper, we introduce MMAC into adaptive Smith predictor to form the multiple model adaptive Smith predictor control method, so as to improve the control performance. According to the possible varying range of the controlled plant's time delay, multiple fixed and adaptive models are established to cover the plant's uncertainties; corresponding controllers are also set up; the switch index function is proposed based on model error to decide the most appropriate controller for the system at every moment and select it as the current controller. Ultimately, multiple adaptive Smith controllers are composed to improve the system's control performance.

The following paper is organized as follows. Section 2 introduces the design principle of Smith predictor control. Section 3 presents the proposed multiple model Smith predictor controller in detail. Numerical simulations are conducted in Section 4 and Section 5 concludes this work. 


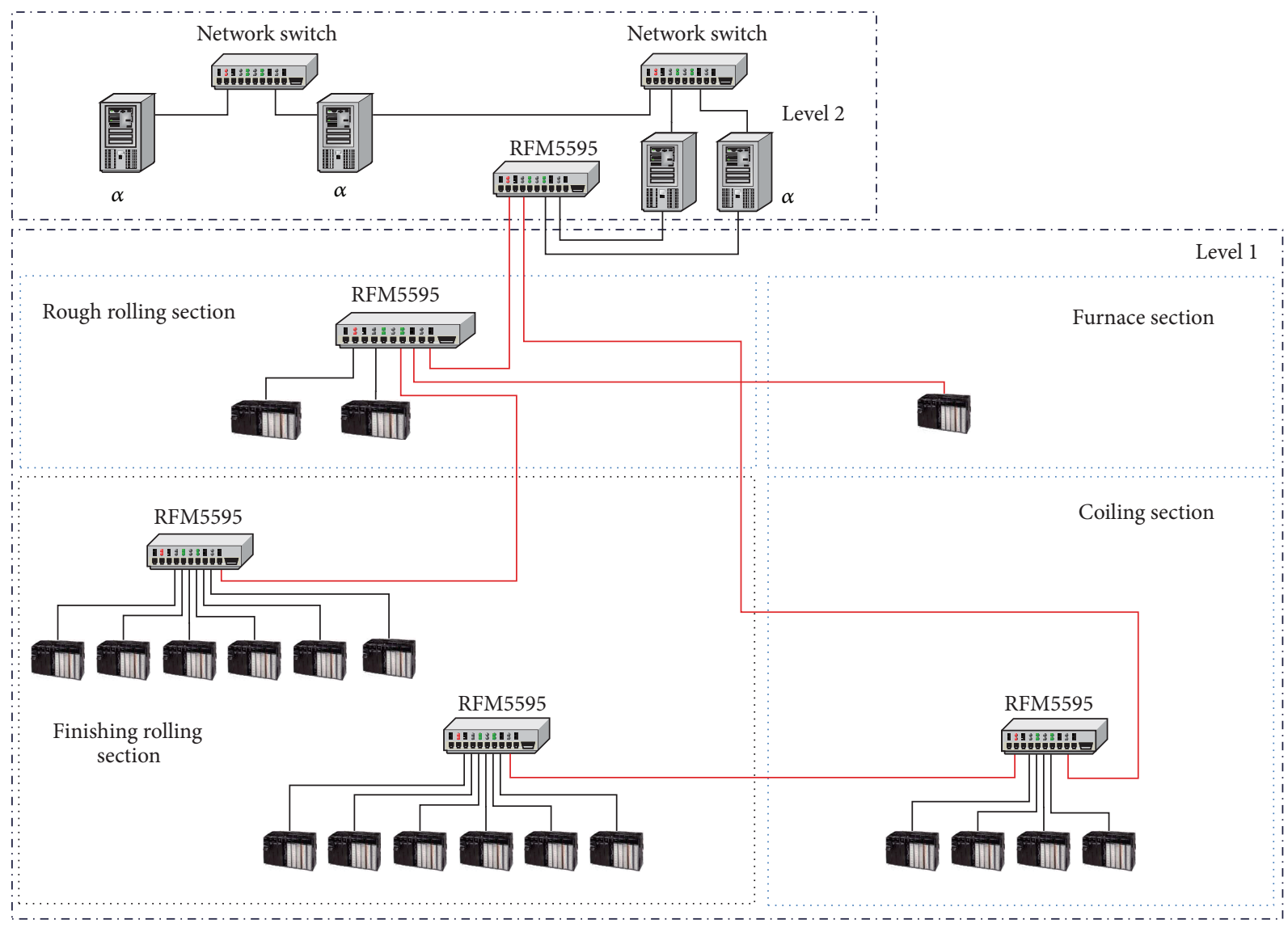

RM Fiber optic network

_ Armored fiber

— Fiber jumpers

Figure 2: RM fiber optic network in a tandem rolling line.

\section{Controller Design Principle Based on Smith Predictor}

2.1. Fixed Smith Predictor Controller. AGC thickness control of rolling mill is a control system with time delay; traditional AGC control methods cannot get satisfying control performance $[13,14]$.

According to control theory, to cope with the timedelay problem, we can take the Smith predictor scheme. A prediction model is added into the AGC process as the feedback loop, which can predict the change of system output and give feedback signal in advance, so as to offset the original system delay and make the characteristic equation of the whole closed loop system without time delay [15]. The principle of Smith predictor in AGC process [16] is shown in Figure 3.

In Figure $3, h_{r}(t)$ and $C(t)$ are the preset value and measured value of the exit thickness, respectively, $\Delta h(t)$ is the thickness difference, $\Delta S$ is the regulation value of the rolling gap, $G_{c}(s)$ is the controller, $G(s) \mathrm{e}^{-\tau s}$ is the transfer function of controlled plant, and $G_{m}(s) \mathrm{e}^{-\tau_{m} s}$ is the prediction model.

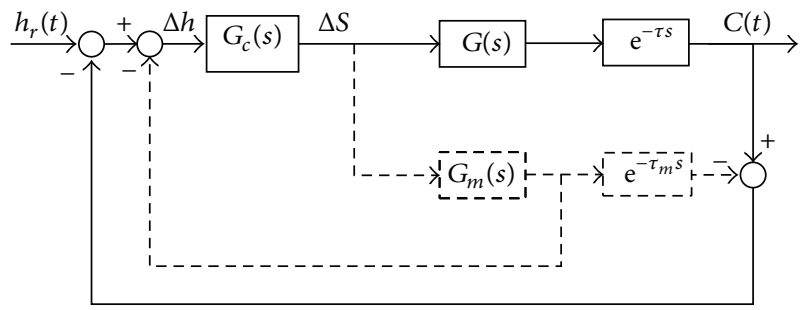

Figure 3: AGC control process based on Smith predictor.

When Smith predictor is not used, the system transfer function is

$$
C(s)=\frac{G_{c}(s) G(s) \mathrm{e}^{-\tau s}}{1+G_{c}(s) G(s) \mathrm{e}^{-\tau s}} h_{r}(s) .
$$

Corresponding characteristic equation is $1+G_{c}(s) G(s)$ $\mathrm{e}^{-\tau s}=0$.

As the pure time-delay part $\mathrm{e}^{-\tau s}$ exists in both transfer function and characteristic equation, with the increase of time delay $\tau$, the phase delay increases, system stability 


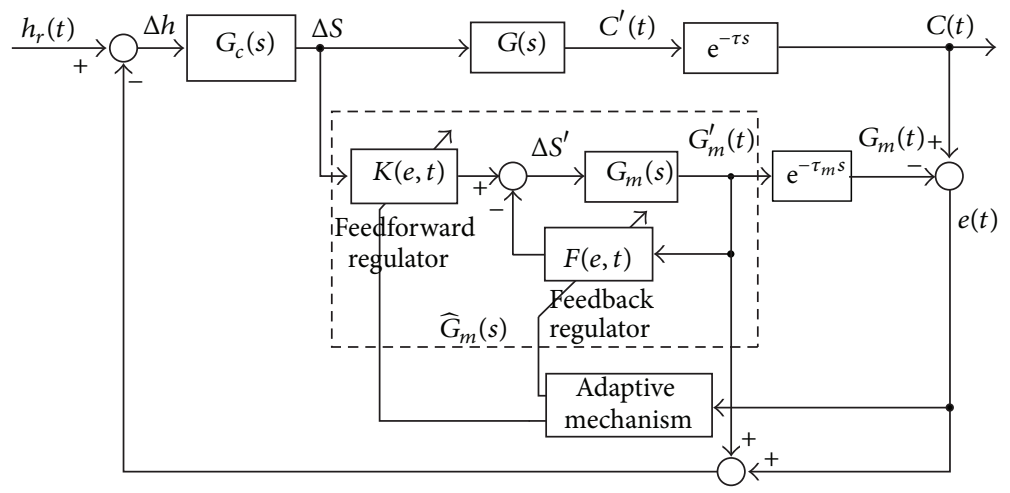

FIGURE 4: AGC control process based on adaptive Smith predictor.

decreases, and the control performance goes worse, while in the Smith predictor scheme, when the prediction model matches the controlled plant perfectly, that is, $G_{m}(s)=G(s)$, $\tau_{m}=\tau$, we have $G_{m}(s) \mathrm{e}^{-\tau_{m} s}=G(s) \mathrm{e}^{-\tau s}$ and system output becomes

$C(s)$

$$
\begin{aligned}
= & \frac{G_{c}(s) G(s) \mathrm{e}^{-\tau s}}{1+G_{c}(s) G(s) \mathrm{e}^{-\tau s}-G_{c}(s) G_{m}(s) \mathrm{e}^{-\tau_{m} s}+G_{c}(s) G_{m}(s)} \\
& \times h_{r}(s) \\
= & \frac{G_{c}(s) G(s) \mathrm{e}^{-\tau s}}{1+G_{c}(s) G_{m}(s)} h_{r}(s) .
\end{aligned}
$$

Corresponding characteristic equation becomes $1+$ $G_{c}(s) G_{m}(s)=0$ without time-delay part, so the system stability will not be influenced by time delay $\tau$.

2.2. Adaptive Smith Predictor Controller. Practically, condition $G_{m}(s)=G(s), \tau_{m}=\tau$ cannot be satisfied easily; [17] proposes a more practical method in which adaptive adjusting law of feedback coefficient $F(t)$ and feedforward coefficient $K(t)$ is applied to guarantee the identification Smith predictor $\widehat{G}_{m}(s)=G(s)$ under the condition $\tau_{m}=\tau=0$. The principle of adaptive Smith predictior is shown in Figure 4.

Consider $\tau_{m}=\tau=0$; the transfer functions of the system and Smith predictor model are given as

$$
G(s)=\frac{k}{s+a}, \quad G_{m}(s)=\frac{k_{m}}{s+a_{m}} .
$$

Corresponding state equations can be got as

$$
\begin{gathered}
\dot{C}(t)=-a C(t)+k \Delta S(t), \\
\dot{C}_{m}(t)=-a_{m} C(t)+k_{m} \Delta S^{\prime}(t) .
\end{gathered}
$$

For given generalized error

$$
e(t)=C(t)-C_{m}(t)
$$

Lynaponov function is set up as

$$
v(t)=\frac{1}{2}\left[k_{m} e(t)^{2}+\frac{1}{\eta_{1}} \psi^{2}(t)+\frac{1}{\eta_{2}} \varphi^{2}(t)\right],
$$

where

$$
\begin{gathered}
\psi(t)=a-a_{m}-k_{m} F(t), \\
\varphi(t)=k-k_{m} K(t) .
\end{gathered}
$$

An adaptive control law

$$
\begin{gathered}
F(t)=-\int \eta_{1} e(t) C_{m}(t)+F(0), \\
K(t)=\int \eta_{2} e(t) \Delta S(t)+K(0)
\end{gathered}
$$

can be used to guarantee $e(t) \rightarrow 0, \psi(t) \rightarrow 0$, and $\varphi(t) \rightarrow 0$ when $t \rightarrow \infty$.

Remark 1. The adaptive parameter identification mechanism can be viewed as a kind of data-driven strategy $[18,19]$. Information about process measurements and initial values is required in the condition of given prediction model. However, unlike pure data-driven methods such as partial least squares (PLS), iterative learning control (ILC), and model free adaptive control which only depend on the process measurements [20], this adaptive law is a model-data integrated method [21] which also needs the relative precise prediction model so that the process information can be fully utilized based on the model to realize the system identification.

From Figure 4, this adaptive law can ensure $\widehat{G}_{m}(s)=G(s)$ (where $\widehat{G}_{m}(s)$ is an identification Smith predictor adjusted by $F, G_{m}$, and $\left.K\right)$; the identification Smith predictor $\widehat{G}_{m}(s)$ can be used. But in practice, both $G_{m}(s)=G(s)$ and $\tau_{m}=\tau=0$ are always very hard to be satisfied, because the precise model of the system cannot be setup, and time delay of the system cannot be measured accurately. So above adaptive Smith predictor is also very difficult to be applied. Due to this situation, the following multiple adaptive Smith prediction model controller will be considered. 


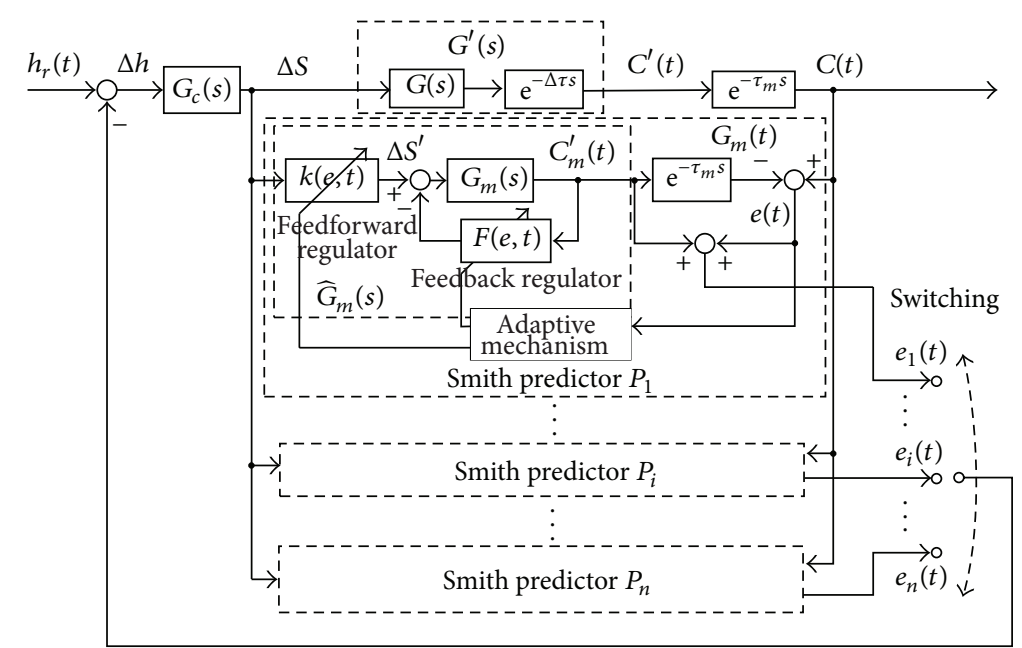

FIGURE 5: AGC control process based on multiple adaptive Smith prediction model.

\section{Multiple Smith Prediction Model Controller}

Consider the rolling system in Figure 5; its transfer function and time delay can be generally described by the following model:

$$
G(s) \mathrm{e}^{-\Delta \tau s} \mathrm{e}^{-\tau_{m} s}
$$

where $\tau_{m}$ is the delay under normal working condition and $\Delta \tau$ stands for the delay fluctuation caused by uncertain factors such as network transmission delay.

Define

$$
G^{\prime}(s)=G(s) \mathrm{e}^{-\Delta \tau s}
$$

Consider the character of first order system; we assume that a first order system with small delay can be approximated by a first order process with different parameters [22]; we have

$$
G^{\prime}(s) \approx \frac{k^{\prime}}{s+a^{\prime}} .
$$

Further, we have the multiple model adaptive control system (see Figure 5).

As in Figure 5, we use the Smith prediction model $\widehat{G}_{m}(s)$ adjusted by $F, G_{m}$, and $K$ to approximate the controlled plant $G^{\prime}(s)$. Consider the varying scope of $\Delta \tau$; parameters of $G^{\prime}(s)$ will be uncertain or time varying, so it is not appropriate to describe the system by single fixed model. For the single adaptive model, if its initial parameters are not properly selected, model parameters will converge slowly. Moreover, if there is drastic system parameter jump, control performance will get worse. So we take multiple models with different initial values to approximate the system model uncertainty.

3.1. Multiple Adaptive Smith Model Controller. Based on the possible parameters variation range of $G^{\prime}(s)$, multiple adaptive Smith predictors $P_{i}(i=1,2, \ldots, n, n$ is the number of models) with different initial values are designed. For original Smith prediction model $G_{m}(s)$, corresponding adaptive parameters of the feedforward and feedback loop satisfy

$$
\begin{gathered}
F_{i}(t)=-\int \eta_{1} e(t) C_{m}^{\prime}(t)+F_{i}(0), \\
K_{i}(t)=\int \eta_{2} e(t) \Delta S(t)+K_{i}(0),
\end{gathered}
$$

where $i=1,2, \ldots, n$.

Define the switch index function

$$
J_{i}\left(t_{0}, t\right)=\alpha e_{i}^{2}(t)+\beta \int_{t_{0}}^{t} e_{i}^{2}(t) d t,
$$

where $i=1,2, \ldots, n, \alpha>0$, and $\beta>0$. And the model error

$$
e_{i}(t)=C^{\prime}(t)-C_{m i}^{\prime}(t) \approx C(t)-C_{m i}(t)
$$

At time $t$, calculate

$$
l\left(t_{0}, t\right)=\underset{i \in\{1,2, \ldots, m\}}{\arg \min } J_{i}\left(t_{0}, t\right)
$$

and Smith predictor based on model $l$ will be switched into the closed loop system.

Remark 2. In practical AGC control process, $C^{\prime}(t)$ cannot be measured, so as in (14), $e_{i}(t)=c(t)-c_{m}(t)$ will be used instead of $C^{\prime}(t)-C_{m}^{\prime}(t)$ in practical control.

3.2. Multiple Fixed Smith Prediction Model Controller. For multiple adaptive Smith prediction model controller, parallel computation of multiple adaptive processes will lead to the increase of calculation amount.

If all the $n$ models are selected to be fixed models, that is, for $i=1,2, \ldots, n$,

$$
\begin{gathered}
F_{i}(t)=F_{i}(0)>0, \\
K_{i}(t)=K_{i}(0)>0 .
\end{gathered}
$$


With enough fixed models, take (13) as the switch index function; calculation amount for multiple models can be decreased drastically. However, without the stable character of adaptive model, system stability can hardly be guaranteed by pure multiple fixed models.

3.3. Multiple Adaptive Smith Controllers with Multiple Fixed and Adaptive Models. Multiple fixed models as in Section 3.2 can reduce the amount of calculation, but due to the lack of adaptive parameter adjusting process, system stability cannot be easily obtained. If in the $n$ models, $n-1$ are set up as fixed models and 1 model is adaptive model, that is, for $i=$ $1,2, \ldots, n-1$,

$$
\begin{gathered}
F_{i}(t)=F_{i}(0)>0, \\
K_{i}(t)=K_{i}(0)>0, \\
F_{n}(t)=-\int \eta_{1} e_{n}(t) C_{m}^{\prime}(t)+F_{n}(0), \\
K_{n}(t)=\int \eta_{2} e_{n}(t) \Delta S(t)+K_{n}(0) .
\end{gathered}
$$

The existence of multiple fixed models reduces the amount of calculation, and the adaptive model guarantees the system stability, so the control performance can be improved as the case of multiple adaptive models.

Theorem 3. If the adaptive Smith controller in Section $2.2(\tau=$ $\left.\tau_{m}=0\right)$ is applied to the AGC system, model error e can be guaranteed to be bounded and to approach zero as $t \rightarrow \infty$; that is, $\lim _{t \rightarrow \infty} e(t)=0$.

Proof. From [17], for adaptive Smith prediction model, taking derivative of Lynaponov function (6), we have

$$
\begin{aligned}
\dot{v}(t)= & -k_{m} a e^{2}(t)+\psi(t)\left[\frac{1}{\eta_{1}} \dot{\psi}(t)-k_{m} e(t) C_{m}(t)\right] \\
& +\varphi(t)\left[\frac{1}{\eta_{2}} \dot{\varphi}(t)+k_{m} e(t) \Delta S(t)\right]
\end{aligned}
$$

from (7) and (8)

$$
\dot{v}(t)=-k_{m} a e^{2}(t) \leq 0 .
$$

Consider $\eta_{1}>0, \eta_{2}>0, k>0$, and $k_{m}>0$, so $v(t)>0$ holds for all $t \neq 0$.

From (19) $v(t)$ is bounded, so $e(t), \psi(t)$, and $\varphi(t)$ are all bounded. Further, we have

$$
\int_{t_{0}}^{t} e^{2}(t) d t<\infty, \quad e(t) \in L_{2} .
$$

For

$$
\begin{gathered}
\dot{C}_{m}^{\prime}(t)=-a_{m} C_{m}^{\prime}(t)+k_{m} \Delta S^{\prime}(t), \\
\Delta S^{\prime}(t)=K(t) \Delta S(t)-F(t) C_{m}^{\prime}(t), \\
\dot{C}^{\prime}(t)=-a C^{\prime}(t)+k \Delta S(t), \\
e(t)=C^{\prime}(t)-C_{m}^{\prime}(t)
\end{gathered}
$$

we have

$$
\dot{e}(t)=-a e(t)-\psi(t) C_{m}^{\prime}(t)+\varphi(t) \Delta S(t) .
$$

Consider $\Delta S(t), C^{\prime}(t)$, and $e(t)$ are bounded; then $C_{m}^{\prime}(t)$ is bounded; from (22) we have $\dot{e}(t) \in L_{\infty}$; from (20) and the Barbalet Lemma [23], we have $\lim _{t \rightarrow \infty} e(t)=0$.

Theorem 4. Take (15) as the switch index function, and select multiple adaptive models in Section 3.1 or multiple fixed models and one adaptive model in Section 3.3 as the multiple adaptive Smith controllers. For the AGC Smith controller shown as in Figure 5, each of these two kinds of controllers can cancel the fluctuation of system delay effectively and improve the control performance.

Proof. (1) For multiple model control composed of multiple adaptive Smith predictors, since each Smith model has its own adaptive mechanism, so for each model we have

$$
\begin{gathered}
J_{i}\left(t_{0}, t\right)=\alpha e_{i}^{2}(t)+\beta \int_{t_{0}}^{t} e_{i}^{2}(t) d t<\infty, \\
i=1,2, \ldots, n, \\
\lim _{t \rightarrow \infty} e_{i}(t)=0 .
\end{gathered}
$$

Though there is model switching among multiple models based on the switch index function, it finally comes to

$$
\Delta S\left(\widehat{G}_{m i}(s)-G^{\prime}(s)\right)=0
$$

Uncertain part of time delay is canceled, and multiple models are used to improve the transient process.

(2) For multiple model control composed of multiple fixed Smith predictors and one adaptive Smith predictor, as to the adaptive Smith predictor

$$
J_{n}\left(t_{0}, t\right)=\alpha e_{n}^{2}(t)+\beta \int_{t_{0}}^{t} e_{n}^{2}(t) d t<\infty .
$$

Consider the switch index function

$$
l\left(t_{0}, t\right)=\underset{i \in\{1,2, \ldots, n\}}{\arg \min } J_{i}\left(t_{0}, t\right) .
$$

For each fixed model

$$
\begin{array}{r}
J_{i}\left(t_{0}, t\right)=\alpha e_{i}^{2}(t)+\beta \int_{t_{0}}^{t} e_{i}^{2}(t) d t, \\
i=1,2, \ldots, n-1 .
\end{array}
$$

If

$$
\lim _{t \rightarrow \infty} J_{i}\left(t_{0}, t\right)=\infty, \quad i=1,2, \ldots, n-1,
$$

then finally the adaptive model will be selected and the adaptive Smith model matches the system model completely; that is, $\Delta S\left(\widehat{G}_{m n}(s)-G^{\prime}(s)\right)=0$. 
If there is a fixed model $i$ satisfying

$$
\lim _{t \rightarrow \infty} J_{i}\left(t_{0}, t\right)=\lim _{t \rightarrow \infty}\left[\alpha e_{i}^{2}(t)+\beta \int_{t_{0}}^{t} e_{i}^{2}(t) d t\right]<\infty,
$$

where $i=1,2, \ldots, n-1$, from $(21)$

$$
\begin{gathered}
\dot{C}^{\prime}(t)=-a C^{\prime}(t)+k \Delta S(t), \\
\dot{C}_{m i}^{\prime}(t)=-\left[a_{m}+k_{m} F_{i}(0)\right] C_{m i}^{\prime}(t)+k_{m} K_{i}(0) \Delta S(t), \\
e_{i}(t)=C^{\prime}(t)-C_{m i}^{\prime}(t) .
\end{gathered}
$$

If the parameters of selected fixed model satisfy $F_{i}(0)>0$ or $a_{m}+k_{m} F_{i}(0)>0$, then $C_{m i}^{\prime}$ is bounded and from (7), (29), and (30), $e_{i}(t), \psi_{i}(t)$, and $\varphi_{i}(t)$ are bounded; the following inequation also holds:

$$
\dot{e}_{i}(t)=-a e_{i}(t)-\psi_{i}(t) C_{m}^{\prime}(t)+\varphi_{i}(t) \Delta S(t)<\infty ;
$$

then from (29) and (31) and the Barbalet Lemma, we have

$$
\begin{gathered}
\lim _{t \rightarrow \infty} e_{j}(t)=0, \\
j \in\left\{j=n, \text { or } j=w \mid \lim _{t \rightarrow \infty} J_{w}\left(t_{0}, t\right)<\infty,\right. \\
w=1,2, \ldots, n-1\}
\end{gathered}
$$

and it finally comes to

$$
\Delta S\left(\widehat{G}_{m j}(s)-G^{\prime}(s)\right)=0 .
$$

The multiple model adaptive Smith predictor still has the matching function.

Remark 5. Multiple model control based on multiple fixed Smith models can improve the transient response, but as it lacks the adaptive parameter regulation, system stability can hardly be guaranteed.

Remark 6. The existence of multiple models covering the parameter uncertain range of the controlled plant can improve the system's transient response and improve the control quality for system with jumping parameter.

\section{Simulation Research}

A first order time delay system as follows will be simulated for rolling AGC system:

$$
\begin{aligned}
G(s) \mathrm{e}^{-\tau s} & =\frac{k}{s+a} \mathrm{e}^{-\tau s}=\frac{0.21}{0.053 s+1} \mathrm{e}^{-0.35 s} \\
& =G^{\prime}(s) \mathrm{e}^{-0.3 s}
\end{aligned}
$$

where $G^{\prime}(s)=(0.21 /(0.053 s+1)) \mathrm{e}^{-0.05 s}$.

The same PID controller as in [17] based on ITAE criterion [24] will also be used; that is,

$$
\begin{aligned}
G_{c}(s) & =K_{p}\left(1+\frac{1}{T_{i} s}+\frac{T_{d} s}{1+T_{d} s / 10}\right) \\
& =2.23\left(1+\frac{1}{0.2 s}+\frac{0.034 s}{0.0034 s+1}\right) .
\end{aligned}
$$

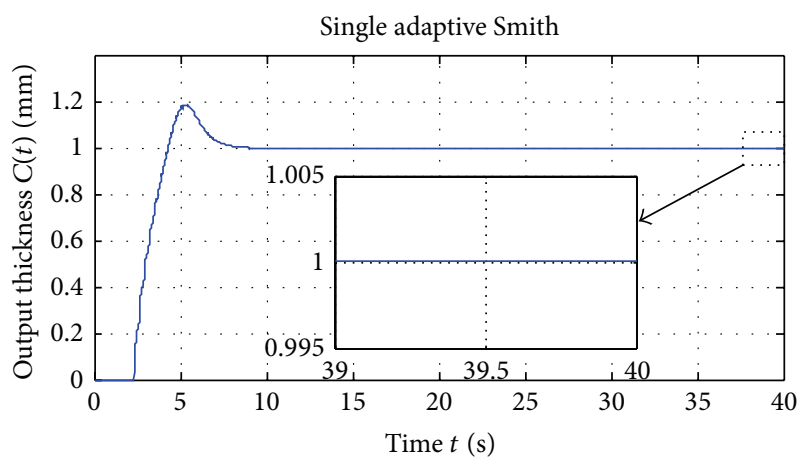

FIGURE 6: Single model adaptive Smith predictor.

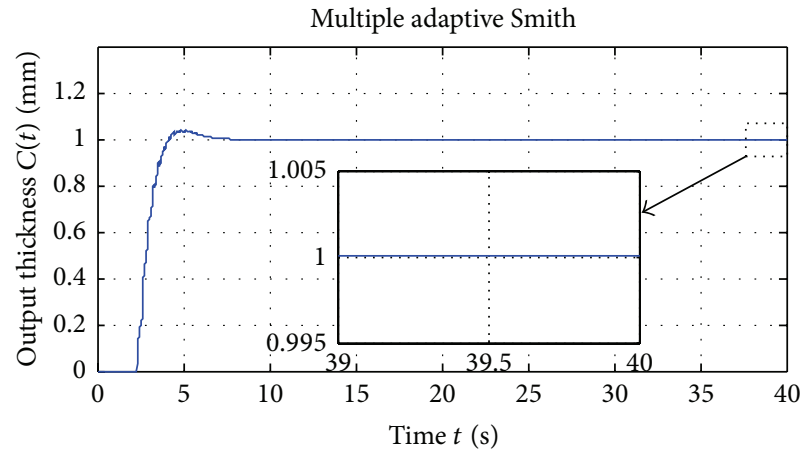

FIGURE 7: Multiple model adaptive Smith predictor.

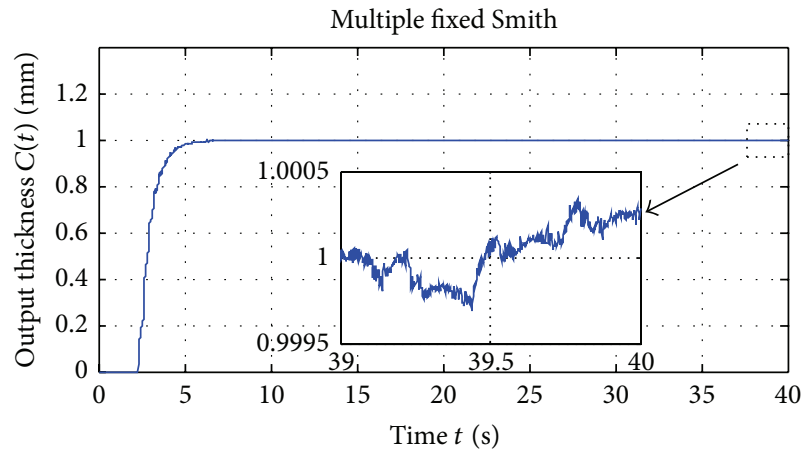

FIGURE 8: Multiple fixed Smith prediction model.

The compensation Smith predictor will be designed as $G_{m}(s)=0.21 /(0.053 s+1), \tau_{m}=0.3 s$. And parameters of $F(t)$ and $K(t)$ will be tuned as (8).

4.1. Multiple Model Smith Predictor for System with Fixed Time Delay. For single adaptive Smith prediction model, let the feedforward and feedback adaptive initial parameters be $F(0)=35$ and $K(0)=50$ in (8); the control result is shown in Figure 6. For multiple model adaptive control, four adaptive Smith prediction models are established, corresponding feedforward and feedback adaptive initial parameters in (12) are $F_{1}(0)=35, F_{2}(0)=30, F_{3}(0)=25, F_{4}(0)=18$, and $K_{1}(0)=$ $K_{2}(0)=K_{3}(0)=K_{4}(0)=50$. The control result is shown in Figure 7. 


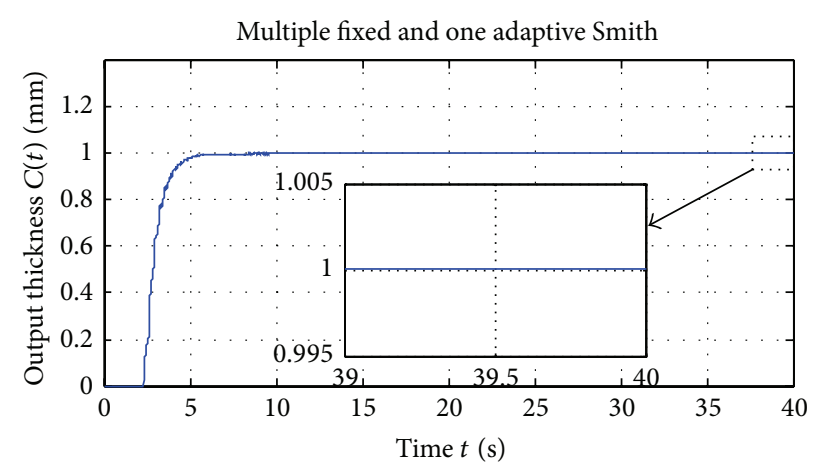

(a) System output

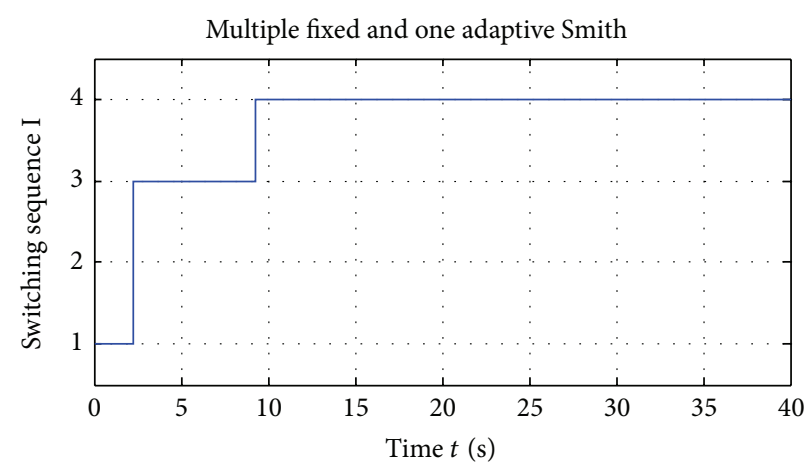

(b) Switching sequence

FIGURE 9: Multiple fixed and single adaptive Smith prediction model.

It can be seen in Figures 6 and 7, when multiple adaptive Smith prediction models with multiple groups of feedforward and feedback adaptive initial parameters are applied to the AGC system, the overshot and system response time are decreased obviously compared with single Smith prediction model case. Meanwhile, when single model adaptive Smith predictor is applied, because model parameters match badly with system parameters, initial identification error is relatively big and the identification time goes relatively long correspondingly. Since multiple model adaptive Smith predictor adopts multiple adaptive initial parameters, system can switch to the closest matching model adaptively, and consequently, system identification error and identification time decrease obviously.

When four fixed Smith prediction models with initial adaptive feedback parameters $F_{1}(0)=35, F_{2}(0)=30$, $F_{3}(0)=25$, and $F_{4}(0)=18$ and same initial feedforward parameters $K_{1}(0)=K_{2}(0)=K_{3}(0)=K_{4}(0)=50$ in (16) are applied, the control result is shown in Figure 8. At the same time, the mixed multiple model Smith predictor with three fixed models whose initial feedback adaptive parameters are $F_{1}(0)=35, F_{2}(0)=30$, and $F_{3}(0)=18$ and an adaptive model with initial value $F_{4}(0)=18$, the same feedforward parameters $K_{1}(0)=K_{2}(0)=K_{3}(0)=K_{4}(0)=50$ are given, and the control results can be seen in Figure 9.

Compared with multiple adaptive Smith predictor model, the computing time can be reduced by using multiple fixed Smith predictor models, but the stability cannot be guaranteed because of the absence of adaptive adjustment. From Figure 8, suitable number of fixed models can be used to improve the overshoot and response time, but the steady identification error cannot be made into 0 at last. In Figure 9, the combination of fixed and adaptive Smith models will give an improved transient response without the loss of final property of system stability. The model switching process is shown in Figure 9(b), the initial fixed model with $F_{1}(0)=35$ is not the best one, and fixed model 3 with $F_{3}(0)=18$ is selected to control the system for a period of time; finally the control model is switched to the 4th adaptive Smith model.

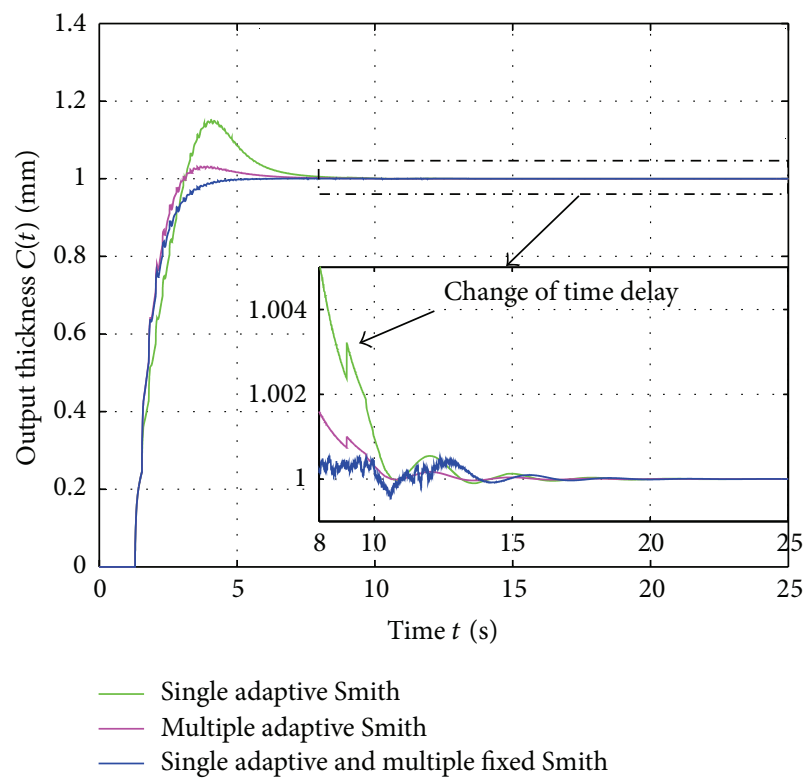

FIGURE 10: Multiple Smith prediction model for system with jumping time delay.

4.2. Multiple Smith Prediction Model for System with Jumping Time Delay. The rolling AGC system with jumping time delay can be described as

$$
\begin{gathered}
G(s) \mathrm{e}^{-\tau s}=\frac{0.21}{0.053 s+1} \mathrm{e}^{-\tau s}, \\
\tau= \begin{cases}0.3 & 0<t<10 \\
0.7 & t \geq 10 .\end{cases}
\end{gathered}
$$

Take the single adaptive Smith prediction model, multiple adaptive Smith prediction model, and the multiple mixed fixed-adaptive Smith prediction model with the same initial feedback and feedforward parameters as in Section 4.1, and we set $\tau_{m}=0.25$. Control results are shown in Figure 10 . 
When system time delay jumps, the present multiple adaptive Smith prediction model and multiple mixed fixedadaptive Smith prediction model can both guarantee the system stability. Meanwhile, compared with single adaptive Smith prediction model, system transient response of multiple adaptive Smith prediction model has been improved obviously no matter before or after the time delay change point.

\section{Conclusion}

In the rolling AGC control process, control systems under complex network condition are generally used. Consider the time delay of network transmission and thickness gauge is usually far away from the roll gap, the transfer function model of the controlled plant will always be described by a first order system with uncertain time delay. Generally, fixed and adaptive Smith predictors are the effective methods to solve this problem, but they all need the precisely known time delay value which is hard to be obtained. This paper approximates the variation of time delay and the parameters in system model by a first order process with different parameters. Multiple Smith prediction model for AGC will be used to set up a MMAC to cope with the change range of time delay in rolling process. The problem of regular Smith predictor is solved and the transient response of adaptive Smith predictor is improved.

\section{Conflict of Interests}

The authors declare that there is no conflict of interests regarding the publication of this paper.

\section{Acknowledgments}

This work was supposed by the Fundamental Research Funds for the Central Universities under Grant FRF-TP-12005B; the Program for New Century Excellent Talents in Universities under Grant NCET-11-0578; and the Specialized Research Fund for the Doctoral Program of Higher Education (SRFDP) under Grant 20130006110008.

\section{References}

[1] W. Yang, Y. Sun, C. Cao, C. Wang, and J. Yang, "Distributed computer control system for hot narrow strip mill," Iron and Steel, vol. 28, no. 8, pp. 30-34, 1993.

[2] Y. Q. Wang, M. H. Sun, and W. Zhang, "Distributed computer control in hydraulic AGC system of tandem cold rolling mill," Machine Tool and Hydraulics, vol. 35, no. 3, pp. 120-123, 2007.

[3] Y. K. Sun, "The computer control system applied to rolling process," Engineering Science, vol. 2, no. 1, pp. 73-76, 2000.

[4] Y. Wang, M. Sun, W. Zhang, Y. Fang, G. Chen, and Q. Zhang, "Research on feedforward control system of tandem cold rolling mill's hydraulic AGC and its computer control strategy," in Proceedings of the International Technology and Innovation Conference (ITIC '06), pp. 2093-2098, Hangzhou, China, November 2006.
[5] D. Q. Song, Research about feedback AGC control stragety of reversible cold rolling mill control systems based on adaptive Smith predictor [Ph.D. thesis], Central South University, Changsha, China, 2009.

[6] C. L. Lai and P. L. Hsu, "Design the remote control system with the time-delay estimator and the adaptive smith predictor," IEEE Transactions on Industrial Informatics, vol. 6, no. 1, pp. 7380, 2010.

[7] K. S. Narendra and J. Balakrishnan, "Improving transient response of adaptive control systems using multiple models and switching," IEEE Transactions on Automatic Control, vol. 39, no. 9, pp. 1861-1866, 1994.

[8] Z. Han and K. S. Narendra, "New concepts in adaptive control using multiple models," IEEE Transactions on Automatic Control, vol. 57, no. 1, pp. 78-89, 2012.

[9] K. S. Narendra and J. Balakrishnan, "Adaptive control using multiple models," IEEE Transactions on Automatic Control, vol. 42, no. 2, pp. 171-187, 1997.

[10] K. S. Narendra and C. Xiang, "Adaptive control of discretetime systems using multiple models," IEEE Transactions on Automatic Control, vol. 45, no. 9, pp. 1669-1686, 2000.

[11] B. Chaudhuri, R. Majumder, and B. C. Pal, "Application of multiple-model adaptive control strategy for robust damping of interarea oscillations in power system," IEEE Transactions on Control Systems Technology, vol. 12, no. 5, pp. 727-736, 2004.

[12] W. T. Chen and B. D. O. Anderson, "A combined multiple model adaptive control scheme and its application to nonlinear systems with nonlinear parameterization," IEEE Transactions on Automatic Control, vol. 57, no. 7, pp. 1778-1782, 2012.

[13] K. Ibrahim, "A new Smith predictor and controller for control of processes with long dead time," ISA Transactions, vol. 42, no. 1, pp. 101-110, 2003.

[14] S.-B. Tan, M.-W. Bao, and J.-C. Liu, "Research on application of smith predictor to monitor AGC in hot strip rolling mills," in Proceedings of the Chinese Control and Decision Conference (CCDC '09), pp. 4172-4175, Guilin, China, June 2009.

[15] J. C. Liu, S. S. Gu, S. F. Zheng, and H. T. Mi, "Application of smith prediction control strategy to AGC system," Iron and Steel, vol. 33, no. 10, pp. 40-43, 1998.

[16] D. Meng, Y. Jia, J. Du, and F. Yu, "Learning control for time-delay systems with iteration-varying uncertainty: a Smith predictorbased approach," IET Control Theory \& Applications, vol. 4, no. 12, pp. 2707-2718, 2010.

[17] X. Li, D. Q. Song, S. Y. Yu, and W. H. Gui, "Feedback automatic gauge control system using model reference adaptive Smith predictor," Control Theory and Applications, vol. 26, no. 9, pp. 999-1003, 2009.

[18] S. Yin, S. X. Ding, X. C. Xie, and H. Luo, "A review on basic datadriven approaches for industrial process monitoring," IEEE Transactions on Industrial Electronics, vol. 61, no. 11, pp. 64186428, 2014.

[19] S. Yin, X. Yang, and H. R. Karimi, "Data-driven adaptive observer for fault diagnosis," Mathematical Problems in Engineering, vol. 2012, Article ID 832836, 21 pages, 2012.

[20] S. Yin, G. Wang, and H. R. Karimi, "Data-driven design of robust fault detection system for wind turbines," Mechatronics, vol. 24, no. 4, pp. 298-306, 2014.

[21] S. Yin, X. W. Li, H. J. Gao, and O. Kaynak, "Data-based techniques focused on modern industry: an overview," IEEE Transactions on Industrial Electronics, 2014. 
[22] S. S. Hu, Automatic Control Theory, Science Press, Beijing, China, 5th edition, 2007.

[23] K. S. Narendra and A. M. Annaswamy, Stable Adaptive Systems, Prentice Hall, Englewood Cliffs, NJ, USA, 1989.

[24] N. Anne and L. Jonathan, "Varying time delay Smith predictor process controller," ISA Transactions, vol. 25, no. 3, pp. 77-81, 2004. 


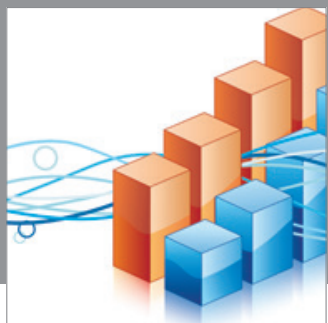

Advances in

Operations Research

mansans

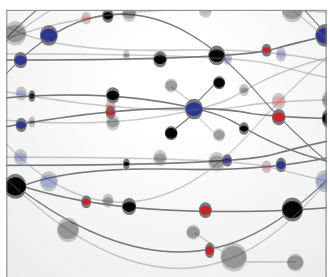

The Scientific World Journal
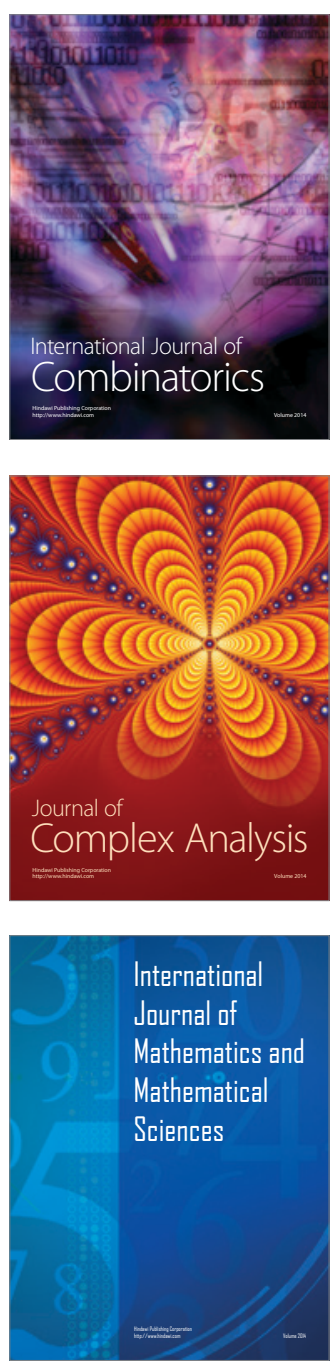
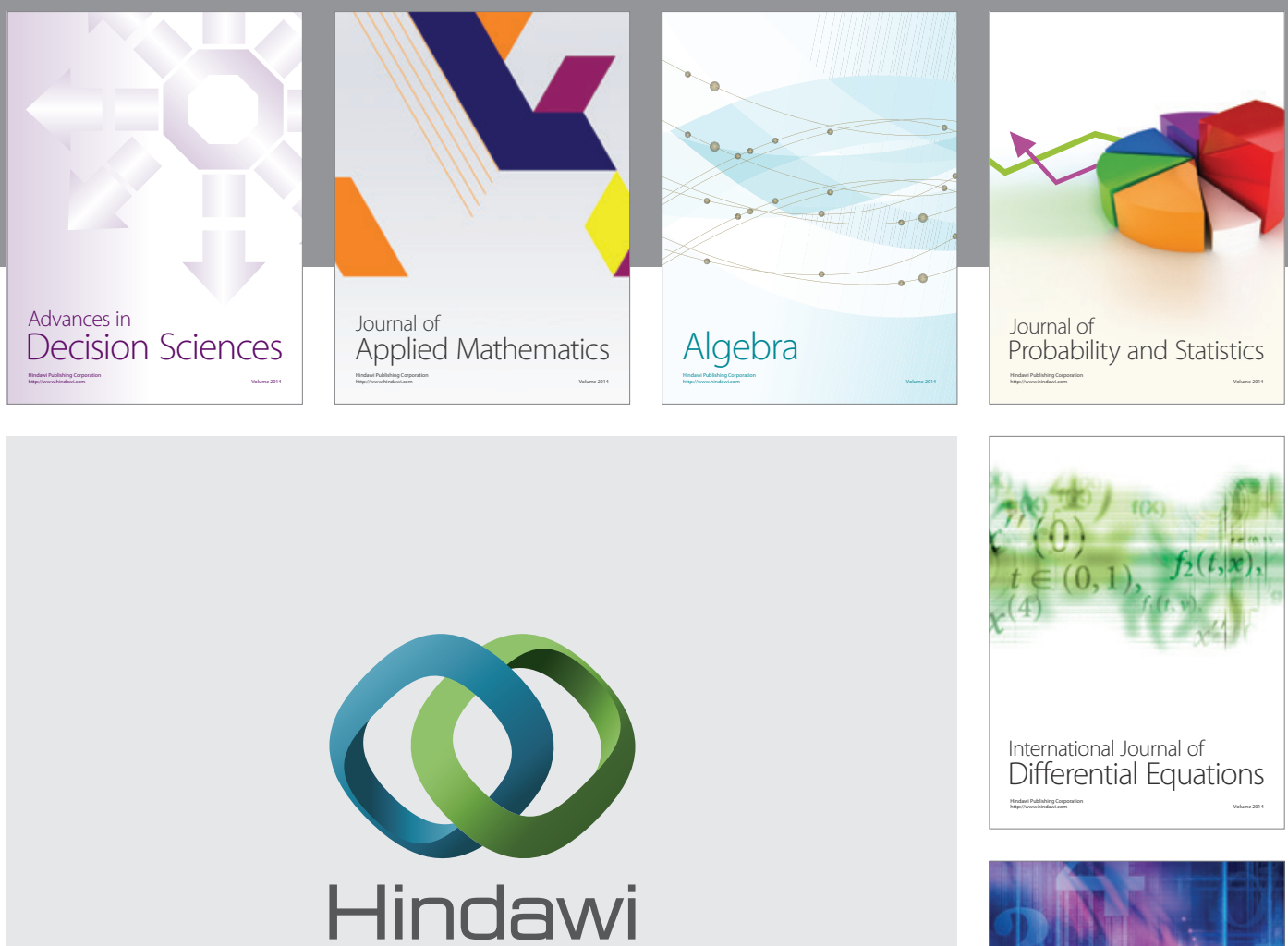

Submit your manuscripts at http://www.hindawi.com
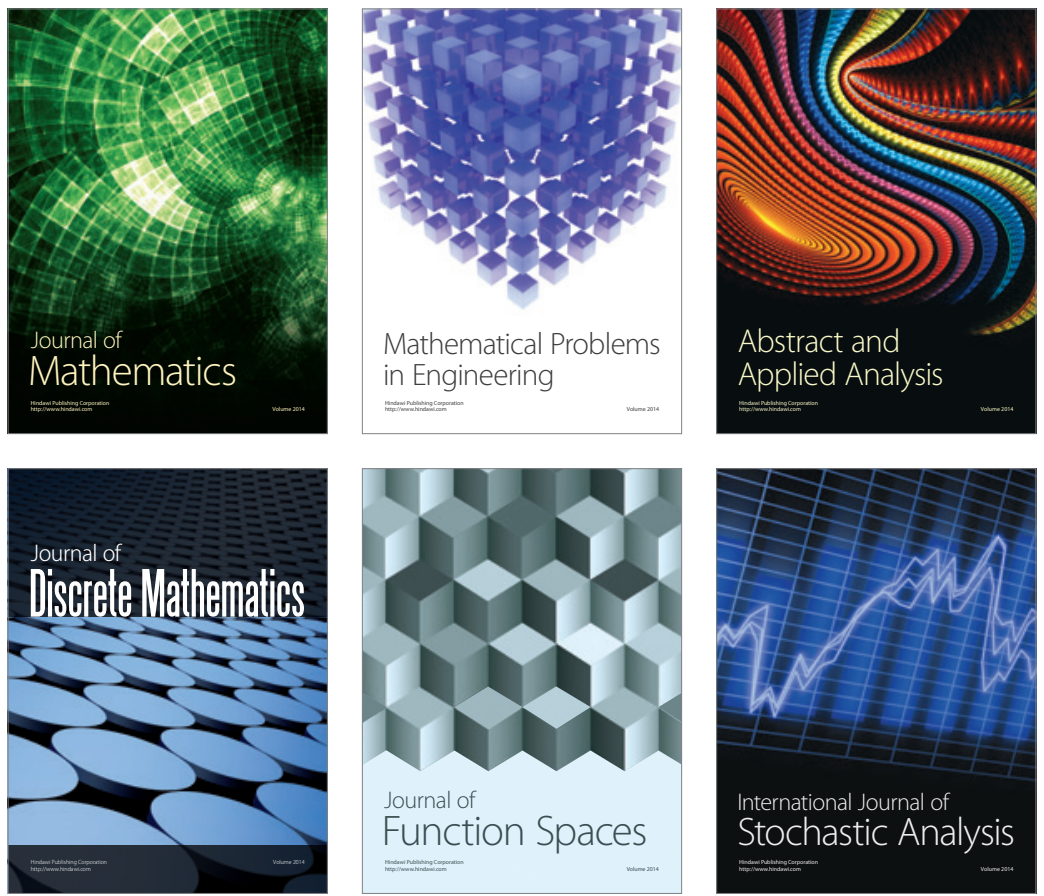

Journal of

Function Spaces

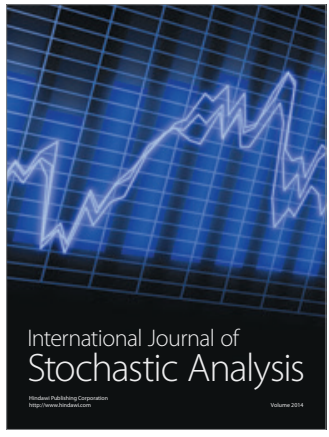

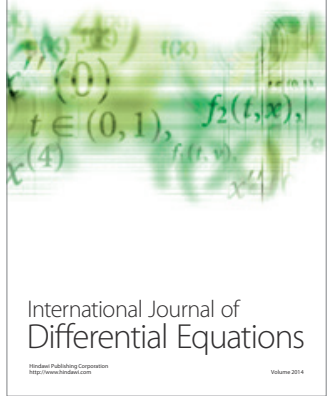
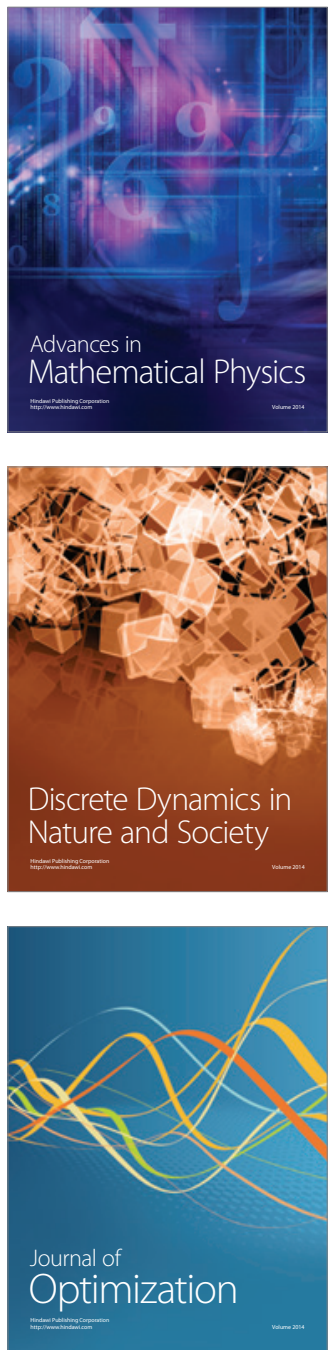